\title{
Factors and outcomes associated with improved left ventricular systolic function in patients with cardiomyopathy
}

\author{
Dylan S. Eiger ${ }^{1}\left(\mathbb{D}\right.$, Lurdes Y.T. Inoue ${ }^{2}$, Qijun $\mathrm{Li}^{2}$, Gust Bardy ${ }^{3}$, Kerry Lee ${ }^{1,4}$, \\ Jeanne Poole ${ }^{5}$, Daniel Mark ${ }^{1,6}$, Zainab Samad ${ }^{1}$, Daniel Friedman ${ }^{1}$, \\ Daniel Fishbein ${ }^{5}$, Gillian Sanders ${ }^{1,6}$, Sana M. Al-Khatib ${ }^{1,6}$ \\ ${ }^{1}$ Department of Medicine, Duke University, Durham, NC, USA \\ ${ }^{2}$ Department of Biostatistics, University of Washington, Seattle, WA, USA \\ ${ }^{3}$ The Seattle Institute for Cardiac Research, Seattle, WA, USA \\ ${ }^{4}$ Department of Biostatistics and Bioinformatics, Duke University, Durham, NC, USA \\ ${ }^{5}$ Department of Medicine, University of Washington, Seattle, WA, USA \\ ${ }^{6}$ Duke Clinical Research Institute, Duke University, Durham, NC, USA
}

\begin{abstract}
Background: Many patients in the Sudden Cardiac Death in Heart Failure Trial (SCD-HeFT) had a significant improvement (>10\%) in the left ventricular ejection fraction (LVEF) during the course of the study, but the factors and outcomes associated with such improvement are uncertain.

Methods: We examined factors and rates of mortality, cause-specific mortality, and implantable cardioverter-defibrillator (ICD) shocks associated with improvement in LVEF by analyzing patients in the SCD-HeFT who were randomized to placebo or an ICD and who had an LVEF checked during follow-up.

Results: During a median follow-up of 3.99 years, of 837 patients who had at least two follow-up LVEF measurements, 276 (33\%) patients had $>10 \%$ improvement in LVEF and 561 (67\%) patients had no significant change in LVEF. Factors significantly associated with LVEF improvement included female sex, white race, history of hypertension, a QRS duration $<120 \mathrm{~ms}$, and beta-blocker use. Improvement in LVEF was associated with a significant improvement in survival. There was no significant association between improvement in LVEF and cause-specific death, but there was a significant association between improvement in LVEF and reduced risk of receiving appropriate ICD shocks.

Conclusions: About a third of patients in this analysis, who were randomized to placebo or an ICD in SCD-HeFT, had a significant improvement in LVEF during follow-up; improvement in LVEF was associated with improved survival but not with cause-specific death, and with decreased likelihood of receiving appropriate ICD shocks. (Cardiol J 2022; 29, 6: 978-984)

Key words: defibrillator, implantable, heart failure, sudden cardiac death, arrhythmia
\end{abstract}

\section{Introduction}

Treatment guidelines for the prevention of sudden cardiac death in patients with heart failure and reduced left ventricular ejection fraction
(LVEF) have been informed by the results of various groundbreaking clinical trials [1-3]. One such trial is the pivotal Sudden Cardiac Death in Heart Failure Trial (SCD-HeFT), which showed a $23 \%$ relative risk reduction in mortality with an implant-

Address for correspondence: Sana Mustapha Al-Khatib, MD, Professor of Medicine, Duke University Medical Center, 7521 N Pavilion Bldg, Durham NC 27715, USA, tel: 919-668-8649, e-mail: alkha001@mc.duke.edu

Received: 1.06.2020 Accepted: 2.12.2020 Early publication date: 31.12 .2020

This article is available in open access under Creative Common Attribution-Non-Commercial-No Derivatives 4.0 International (CC BY-NC-ND 4.0) license, allowing to download articles and share them with others as long as they credit the authors and the publisher, but without permission to change them in any way or use them commercially. 
able cardioverter-defibrillator (ICD) in patients with New York Heart Association (NYHA) class II or III heart failure and LVEF $\leq 35 \%$ despite optimal medical therapy [2]. However, there is a subset of patients who receive an ICD for these criteria who later have improvement in LVEF to $>35 \%$. The factors and outcomes associated with this improvement are not completely understood. A prior study showed that patients with heart failure with reduced ejection fraction, who later demonstrate recovery of LVEF above $40 \%$ have a lower rate of all-cause mortality and fewer hospitalizations than patients with heart failure with preserved ejection fraction ( $\mathrm{HFpEF}$ ) or heart failure with a persistently reduced ejection fraction (HFrEF) [4]. These findings suggest that an improved LVEF, even if it remains below normal, is associated with increased survival in patients with heart failure. However, this study was limited by the single-center retrospective study design. The SCD-HeFT overcomes these limitations by being a randomized clinical trial and by enrolling and following patients with a reduced LVEF from multiple medical centers. In this study, we aimed to examine factors and outcomes associated with a significant improvement in LVEF (> 10\%) in SCD-HeFT. This magnitude in LVEF improvement was chosen at the inception of this analysis and was based on clinical judgment of what is considered meaningful LVEF improvement that is not due to errors in measuring LVEF while preserving a reasonable sample size.

\section{Methods}

Briefly, SCD-HeFT enrolled 2521 patients with NYHA class II or III heart failure due to ischemic or non-ischemic cardiomyopathy and an LVEF of $\leq 35 \%$, who were randomly assigned to placebo $(\mathrm{n}=847)$, amiodarone $(\mathrm{n}=845)$, or a shock only, single-lead ICD $(n=829)$. All patients received optimal medical therapy at the time for heart failure. Patients were enrolled in SCD-HeFT from September 16, 1997, to July 18, 2001 and all patients were followed until October 31, 2003.

We selected patients from SCD-HeFT who were in the placebo or ICD arms and had at least three recorded measurements of LVEF during the course of the study with one measurement taken at baseline and at least two measurements obtained during follow-up. These follow-up LVEF measurements were performed ad hoc and were not mandated per the SCD-HeFT protocol. We required patients to have two follow-up LVEF measurements to decrease variability in these measurements and the likelihood of mis-readings.
Patients in the amiodarone arm were excluded from this analysis due to the difficulty to adequately adjust for the confounding factor that amiodarone may have on our results. In addition, prior studies have shown increased risk of mortality with amiodarone, especially in patients with NYHA class III symptoms $[2,5]$.

While for the primary analysis we defined LVEF improvement as $>10 \%$, we considered another definition based on a recent report from the Journal of the American College of Cardiology (JACC) Scientific Expert Panel on Heart Failure with Recovered Left Ejection Fraction, which provided a working definition of heart failure with recovered LVEF as: (1) documentation of a decreased LVEF $<40 \%$ at baseline, (2) $\geq 10 \%$ absolute improvement in LVEF, and (3) a second measurement of LVEF > 40\% [6].

In this analysis, the main outcome was all-cause mortality. Other outcomes of interest included cause-specific mortality and appropriate ICD shocks. Deaths were classified as cardiac or non-cardiac death, and when the event was cardiac, it was further classified as arrhythmic cardiac death, which includes death due to ventricular tachyarrhythmia or bradyarrhythmia, and non-arrhythmic cardiac death, which includes heart failure, non-arrhythmic non-heart failure, and other cardiac causes [7].

Per the SCD-HeFT protocol, the modality to measure LVEF at baseline and during follow-up was not specified. In the majority of patients, the baseline LVEF was measured by an echocardiogram $(n=1461)$, while the remainder were measured by contrast angiography $(n=436)$ and radionuclide angiography $(n=616)$. While granular data on the modality used to measure follow-up LVEF were not available, the vast majority of LVEF assessments during follow-up were done by echocardiography.

For descriptive analyses, we fit a linear mixed-effects model for the log-transformed LVEF and used the estimated slopes to stratify patients into groups consisting of patients with an increase in LVEF by $>10 \%$ vs. not. We log-transformed LVEF measurements and plotted the transformed measurements for each patient against time, and a best-fit line was obtained. The slope of the line was used to assess the overall change in LVEF over time, even if the LVEF initially decreased and then increased, or vice versa, which was then used to determine whether the patients showed $>10 \%$ or $\leq 10 \%$ improvement.

We compared these LVEF groups with descriptive statistics, with means and standard deviations 
for continuous variables and counts and proportions for the categorical variables. In unadjusted analyses, we obtained Kaplan-Meier survival curves comparing the two LVEF groups and examined the mode of death with cumulative incidence functions.

In adjusted analyses, to examine all-cause mortality, we used joint longitudinal-survival models [8-10]. Such models have two components: a model for the longitudinal LVEF trajectory and a model for the survival data that incorporates features of the longitudinal LVEF model as predictors. With joint models, we note that the regression parameters for both the time-to-event and longitudinal outcomes are estimated jointly, unlike the procedure adopted for the descriptive analysis. Specifically, we modeled the log-transformed LVEF as a linear mixed-effects model regressed on time since randomization and baseline variables, and we used a Weibull proportional hazards survival regression model for time to death from all causes regressed on the subject-specific intercept and slope, dichotomized based on $>10 \%$ improvement in LVEF versus $\leq 10 \%$ improvement, from the longitudinal LVEF model and baseline variables including treatment assignment, age, sex, race, history of hypertension, diabetes, pulmonary disease, smoking, ischemic heart disease, prior myocardial infarction (MI), prior coronary artery bypass grafting $(\mathrm{CABG})$, prior percutaneous coronary intervention (PCI), NYHA class of heart failure, QRS duration, baseline LVEF, and treatment with beta-blockers, diuretics, or angiotensin-converting enzyme inhibitors (ACEI).

To examine cause-specific mortality, we utilized joint longitudinal competing risks survival models, which were fit under the Bayesian approach using relatively non-informative priors with JAGS and the R-package rjags.

To examine the risk of appropriate ICD therapy, we fitted a mixed-effects model for the logtransformed LVEF regressed on time since randomization and adjusted for baseline covariates, and a logistic regression model for the outcome of appropriate shocks regressed on the subjectspecific intercept and slope, dichotomized based on $>10 \%$ improvement in LVEF vs. $\leq 10 \%$ improvement. The variables included in this model were sex, age, race, presence or absence of ischemic heart disease, QRS duration, NYHA class of heart failure, history of smoking, diabetes, hypertension, pulmonary disease, prior MI, CABG, or PCI, and treatment with beta-blockers or ACEI. Patients who were classified as having received appropriate shock therapy were those who had documented evidence of appropriate shock therapy, in the pres- ence or absence of other inappropriately delivered ICD therapy. Patients not in this group included those who received only inappropriately delivered shock therapy or no therapy at all.

All analyses were repeated in the subset of patients $(n=248)$ with at least one follow-up LVEF measurement of $>40 \%$.

\section{Results}

Baseline characteristics of patients included ( $\mathrm{n}=837)$ and excluded $(\mathrm{n}=839)$ from our analysis due to having fewer than three LVEF measurements are shown in Supplementary Table 1. Compared with patients who were excluded from the analysis, those who were included were more likely to be white and to have left bundle branch block and a higher baseline LVEF (25\% vs. $23 \%$ ). The included patients had lower rates of hypertension, diabetes, pulmonary disease, and ischemic heart disease, as well as lower baseline blood urea nitrogen and creatinine.

During a median follow-up of 3.99 years, 837 patients had at least one initial and two follow-up LVEF measurements. The average time to first follow-up LVEF was $1.02 \pm 0.28$ years, second follow-up was $2.39 \pm 0.57$ years, and third follow-up was $3.04 \pm 0.98$ years. The median follow-up times for patients included in this study were similar between patients with or without $>10 \%$ improvement in LVEF (4.04 vs. 3.97 years, respectively). Of these patients, 276 (33\% of all patients, of whom 149 had an ICD) had a $10 \%$ improvement in LVEF and 561 (67\% of all patients, of whom 267 had an ICD) had no significant change in LVEF. Of the 276 patients who demonstrated an improvement in LVEF $>10 \%$, only 10 had a final LVEF that remained below $35 \%$. The baseline characteristics of the patients in the two groups are listed in Table 1.

In the adjusted longitudinal model, there was a significant association between improvement in LVEF and female sex $(\mathrm{p}=0.003,1.09,95 \%$ confidence interval [CI] 1.03-1.14), white race ( $p<0.001,1.13,95 \%$ CI 1.08-1.19), history of hypertension ( $\mathrm{p}<0.001,1.10,95 \%$ CI 1.05-1.14), a QRS duration $<120 \mathrm{~ms}$ ( $\mathrm{p}<0.001,1.16,95 \% \mathrm{CI}$ $1.12-1.20)$, and beta-blocker use ( $\mathrm{p}=0.01,1.05$, 95\% CI 1.01-1.10). Notably, the type of cardiomyopathy (ischemic vs. non-ischemic) and prior coronary interventions were not associated with LVEF improvement.

In the adjusted survival model, improvement in LVEF was associated with a significant improvement in survival (hazard ratio [HR] $0.58,95 \% \mathrm{CI}$ 
Table 1. Baseline characteristics between patient groups.

\begin{tabular}{|c|c|c|c|c|c|}
\hline & \multicolumn{2}{|c|}{$\begin{array}{c}\text { No change in LVEF } \\
(\mathrm{n}=561)\end{array}$} & \multicolumn{2}{|c|}{$\begin{array}{c}\text { Increase in LVEF > }>10 \% \\
(n=276)\end{array}$} & \multirow[t]{2}{*}{$\mathbf{P}$} \\
\hline & Mean/Count & SD/Percentage & Mean/Count & SD/Percentage & \\
\hline ICD & 267 & 47.59 & 149 & 53.99 & 0.10 \\
\hline Age & 59 & 16 & 58 & 16 & 0.50 \\
\hline Male & 451 & 80.39 & 186 & 67.39 & $<0.001$ \\
\hline White & 460 & 82 & 223 & 80.8 & 0.74 \\
\hline History of hypertension & 285 & 50.8 & 153 & 55.43 & 0.23 \\
\hline History of diabetes & 161 & 28.7 & 81 & 29.35 & 0.91 \\
\hline History of pulmonary disease & 91 & 16.22 & 51 & 18.48 & 0.47 \\
\hline History of smoking & 428 & 76.29 & 193 & 69.93 & 0.06 \\
\hline eGFR $(0,60)[\mathrm{mL} / \mathrm{min}]$ & 175 & 31.36 & 72 & 26.18 & 0.14 \\
\hline eGFR $\geq 60 \mathrm{~mL} / \mathrm{min}$ & 383 & 68.64 & 203 & 73.82 & - \\
\hline Ischemic disease & 309 & 55.08 & 115 & 41.67 & $<0.001$ \\
\hline Prior CABG & 153 & 27.27 & 59 & 21.38 & 0.08 \\
\hline Prior MI & 275 & 49.02 & 97 & 35.14 & $<0.001$ \\
\hline Time MI (days)* & 1652 & 2977.5 & 1510 & 2670 & 0.47 \\
\hline Prior $\mathrm{PCl}$ & 126 & 22.46 & 42 & 15.22 & 0.02 \\
\hline History of syncope & 0 & 0 & 0 & 0 & NA \\
\hline LVEF [\%] & 24 & 6 & 25 & 7 & 0.04 \\
\hline New York Heart Association 2 & 403 & 71.84 & 204 & 73.91 & 0.58 \\
\hline New York Heart Association 3 & 158 & 28.16 & 72 & 26.09 & - \\
\hline Anti-arrhythmic medication & 0 & 0 & 0 & 0 & NA \\
\hline Beta-blockers & 382 & 68.09 & 186 & 67.39 & 0.90 \\
\hline $\mathrm{ACEl}^{*}$ & 544 & 96.97 & 268 & 97.1 & 1.00 \\
\hline Diuretics & 475 & 84.67 & 233 & 84.42 & 1.00 \\
\hline QRS duration [ms] & 118 & 52 & 104 & 32 & $<0.001$ \\
\hline Right bundle branch block & 30 & 5.35 & 13 & 4.71 & 0.82 \\
\hline Left bundle branch block & 151 & 26.92 & 52 & 18.84 & 0.01 \\
\hline
\end{tabular}

*Time MI: time from the most recent myocardial infarction to enrollment in SCD-HeFT; ACEI — angiotensin-converting enzyme inhibitor; CABG - coronary artery bypass grafting; eGFR - estimated glomerular filtration rate; ICD - implantable cardioverter-defibrillator; $\mathrm{LVEF}$ - left ventricular ejection fraction; $\mathrm{MI}$ - myocardial infarction; $\mathrm{PCl}$ - percutaneous coronary intervention

0.35-0.96). Additionally, there was a significant negative association between baseline LVEF and all-cause mortality. Specifically, patients with a $10 \%$ higher baseline LVEF had a lower mortality risk (HR 0.87, 95\% CI 0.84-0.91). Importantly, ICD use was significantly associated with improved survival independently of change in LVEF and other clinical variables (HR 0.62, 95\% CI 0.43-0.91).

Kaplan-Meier survival curves for the two groups of interest are shown in Figure 1. At 5 years, the survival probability for patients with $>10 \%$ improvement in LVEF was 0.90 (95\% CI 0.85-0.95) while the survival probability for patients with no change in LVEF was 0.77 (95\% CI 0.72-0.81), demonstrating that $>10 \%$ improvement in LVEF is associated with improved survival outcomes.

In the adjusted survival model, there was a significant negative association between baseline LVEF and rates of non-cardiac death, non-arrhythmic cardiac death, and arrhythmic cardiac death. Specifically, patients with a $10 \%$ higher baseline LVEF had lower rates of non-cardiac death (HR 0.95, 95\% CI 0.91-0.98), non-arrhythmic cardiac death (HR 0.91, 95\% CI 0.88-0.94), and arrhythmic cardiac death (HR 0.95, 95\% CI 0.92-0.99). However, there was no association between improvement in LVEF and non-cardiac death (HR 0.92, 95\% CI 0.61-1.41), non-arrhythmic cardiac death (HR 0.73 , 


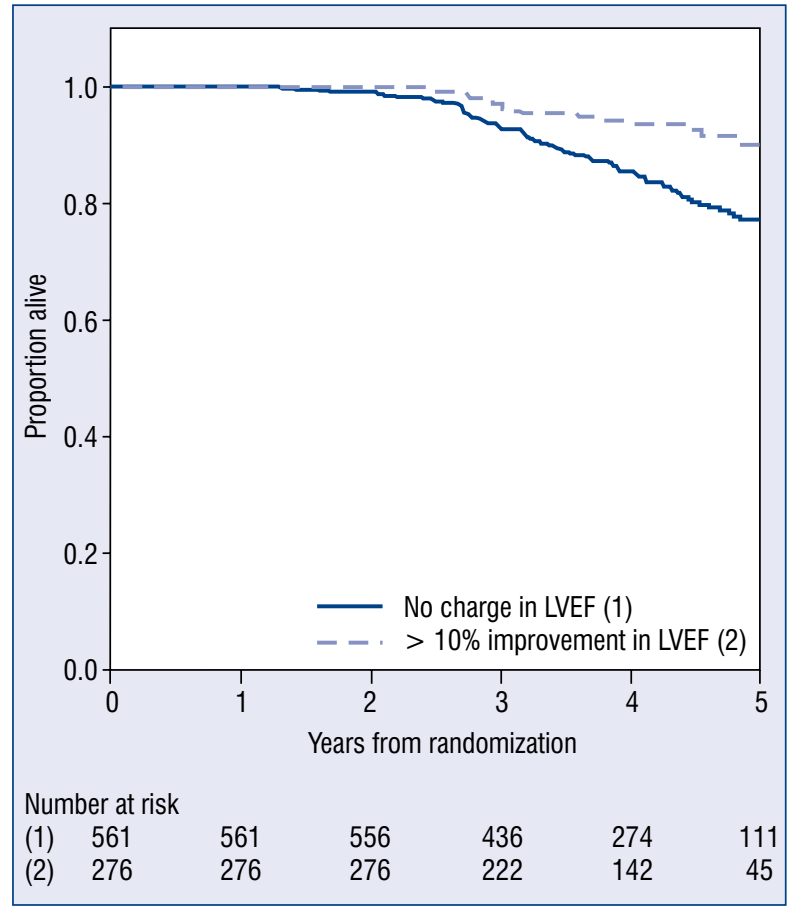

Figure 1. Kaplan-Meier survival curves for groups defined by left ventricular ejection fraction (LVEF). The number of patients at risk for each year in each LVEF category since randomization is listed below the KaplanMeier curve.

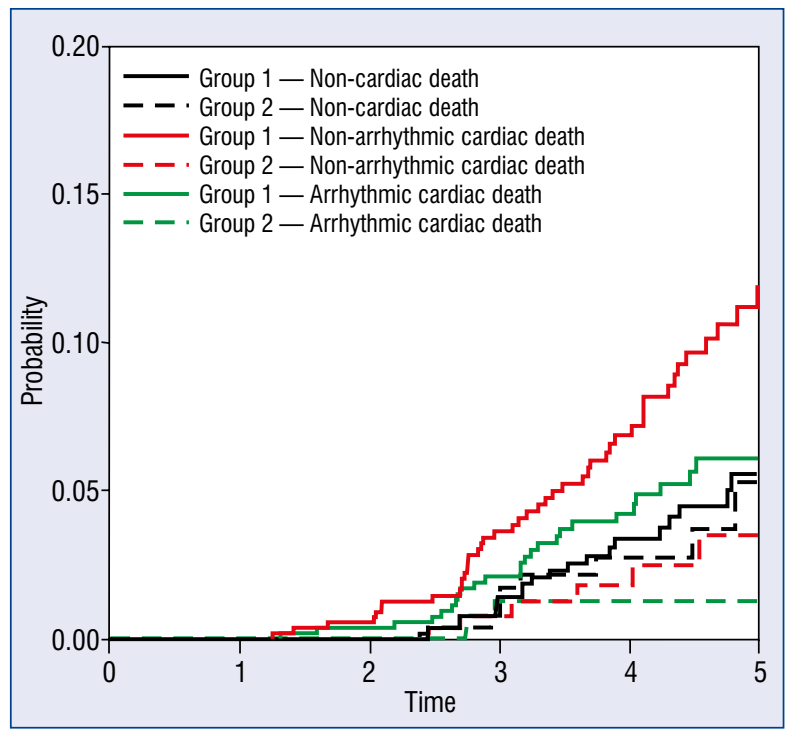

Figure 2. Cumulative incidence functions of cause-specific mode of death for left ventricular ejection fraction (LVEF) groups. Group 1 (solid lines) refers to patients who experienced no change ( $\leq 10 \%$ improvement) in LVEF while Group 2 (dashed lines) refers to patients who demonstrated an improvement of $>10 \%$ in LVEF.
95\% CI 0.49-1.10), and arrhythmic cardiac death (HR 0.84, 95\% CI 0.55-1.27). Cumulative incidence functions for cause-specific death between LVEF groups are shown in Figure 2.

Of 416 patients with an ICD, 96 received appropriate ICD therapy. There was a significant association between improvement in LVEF $>10 \%$ and decreased likelihood of receiving appropriate ICD shocks (odds ratio [OR] 0.27, 95\% CI 0.08-0.77). However, there was no association between baseline LVEF and likelihood of receiving appropriate ICD shocks (OR 0.76, 95\% CI 0.33-1.51).

Repeating all analyses in the subset of patients with at least one follow-up LVEF measurement of $>40 \%(\mathrm{n}=248,206 \mathrm{had}>10 \%$ improvement in LVEF and 42 had $\leq 10 \%$ improvement in LVEF) showed an association between baseline LVEF and survival. However, there was no significant association between improvement in LVEF and overall survival, cause-specific death, and appropriate ICD shocks.

\section{Discussion}

This study demonstrates that a third of patients included in this analysis had $>10 \%$ improvement in LVEF, and factors associated with this improvement are female sex, white race, QRS $<120 \mathrm{~ms}$, history of hypertension, and beta-blocker usage. Additionally, we demonstrated that $>10 \%$ improvement in LVEF was associated with lower risk of all-cause mortality and decreased likelihood of receiving appropriate ICD therapy.

There is a growing body of evidence demonstrating that patients with heart failure with recovered or significantly improved LVEF have a distinct clinical phenotype and physiology that are not properly captured or addressed in clinical trials [11-16]. Improve the Use of Evidence-Based Heart Failure Therapies in the Outpatient Setting (IMPROVE HF) found that patients who had a $>10 \%$ improvement in LVEF were more likely to be women, have a non-ischemic etiology of heart failure, have no prior MI, have a lower ejction fraction at baseline, and not be taking digoxin [14]. While both studies determined that factors such as age, diabetes, and renal insufficiency were not associated with LVEF improvement, some baseline patient characteristics associated with improved LVEF found in IMPROVE HF are not concordant with those found in our study, demonstrating the need for more investigations of this patient population. Prior studies have shown that patients with improved LVEF have improved survival and lower 
risk of all-cause, cardiovascular, and heart failure-related hospitalizations, cardiac transplantation, or left ventricular assist device implantation when compared with patients with $\mathrm{HFrEF}[4,14,17]$. Additionally, in a post hoc analysis of SCD-HeFT by Adabag et al. [18], patients with an improved LVEF to $>35 \%$ had a similar survival benefit from an ICD to those who did not demonstrate this improvement. It is noteworthy that using an LVEF of $>35 \%$ as a definition for LVEF improvement does not normalize the magnitude of change across patients, which Adabag et al. [18] acknowledged as a limitation. For example, a patient with LVEF improvement from $34 \%$ to $36 \%$ and a patient with improvement from $18 \%$ to $36 \%$ were analyzed as being the same by Adabag et al. [18]. We believe that our inclusion criterion of at least two follow-up LVEF measurements and our definition of a clinically significant improvement in LVEF as $>10 \%$ increase the likelihood that the measured change in LVEF and associated findings are real and clinically meaningful. Therefore, our analysis adds to prior studies by using a better definition of LVEF improvement, further identifying factors associated with LVEF improvement, and demonstrating that LVEF improvement is associated with a decreased likelihood of receiving appropriate ICD shocks.

Left ventricular ejection fraction improvement in patients with a primary prevention ICD presents a dilemma to clinicians and patients when deciding to replace the ICD generator at the end of battery life [15, 19-22]. Naksuk et al. [23] demonstrated that patients who had an LVEF improvement to $>35 \%$ and an increase in $>10 \%$ from baseline LVEF had a similar survival benefit before and after ICD replacement when compared with patients who had no improvement in LVEF. Our results support these findings by showing that ICD use was significantly associated with improved survival independent of change in LVEF and other clinical variables.

Whether LVEF improvement is associated with appropriate ICD therapies was examined by Schliamser et al. [24], who determined that while improvement in LVEF was associated with improved survival, it was not with a significant decrease in appropriate ICD shocks. These authors determined that rates of appropriate ICD therapy are similar between those with and without improvement in LVEF while we present evidence for a decreased incidence of receiving appropriate ICD therapy with improvement in LVEF. Although patients with $>10 \%$ improvement in LVEF in our study experienced a lower incidence of appropriate ICD therapy, there was still a clear survival benefit to having an ICD; therefore, this finding does not justify forgoing ICD generator replacement at the end of battery life.

\section{Limitations of the study}

There are several limitations to this analysis. This was a retrospective analysis of prospectively collected data; therefore, we cannot rule out residual confounding factors and selection bias. We were not able to examine subgroups of interest like patients who experience an improvement of $>10 \%$ yet remain beneath an $\mathrm{LVEF}$ of $35 \%$ (10 out of 276 patients).

Follow-up LVEF assessment was not protocol driven, potentially leading to other sources of bias. Also, the observed changes in LVEF, read at enrolling centers and not at a central core facility, may partially be the result of differences in measurement technique or user variability. Approximately $50 \%$ of patients included in this analysis had ischemic heart disease, and data regarding the revascularization procedures conducted during follow-up were unavailable. Additionally, specific data regarding heart rhythm, therapeutic procedures, and other drugs including digoxin, angiotensin receptor blockers, and aldosterone antagonists were also unavailable. Some may critique our definition of an improvement in LVEF. We note that the $>10 \%$ improvement in LVEF was chosen at the inception of this analysis because it was thought to be less prone to errors in reading (e.g. from choosing a 5\% change). In fact, our analysis provides evidence that a $>10 \%$ change in LVEF is associated with changes in clinical outcomes such as mortality and rates of appropriate ICD therapy. Patients included in this analysis had at least two follow-up LVEF measurements, which may have introduced selection-bias against patients who died prior to having a repeat LVEF measurements, and it may reflect differences in overall quality of care. Although repeating the analyses in the subset of patients with at least one follow-up LVEF $>40 \%$ showed that there was no significant association between improvement in LVEF and overall survival, cause-specific death, and appropriate ICD shocks, these analyses were limited by the small sample size. Larger studies are needed to examine the association between LVEF improvement and outcomes in patients with at least one follow-up LVEF $>40 \%$. Finally, optimal medical therapy of heart failure has significantly changed since the completion of SCD-HeFT, which may further influence factors that predict LVEF improvement and limit the applicability of these findings to heart failure patients today. 


\section{Conclusions}

About a third of patients in this analysis, who were randomized to placebo or an ICD in SCD-HeFT, had $>10 \%$ improvement in LVEF during follow-up. Improvement in LVEF was associated with added survival benefit and decreased likelihood of receiving appropriate ICD shocks; however, there was no association with decreased risk of non-cardiac death and arrhythmic- and non-arrhythmic-related cardiac death. While female sex, white race, QRS $<120 \mathrm{ms,}$ history of hypertension, and beta-blocker usage are associated with LVEF improvement, future studies should determine whether additional factors and tests can improve the prediction of LVEF improvement and associated outcomes.

\section{Acknowledgments}

This work was supported by the Duke University Medical Scientist Training Program (to D.S.E.) and the National Institute of General Medical Sciences at the National Institutes of Health (5T32GM007171-45 to D.S.E.).

\section{Conflict of interest: None declared}

\section{References}

1. Al-Khatib SM, Stevenson WG, Ackerman MJ, et al. 2017 AHA/ /ACC/HRS Guideline for Management of Patients With Ventricular Arrhythmias and the Prevention of Sudden Cardiac Death: A Report of the American College of Cardiology/American Heart Association Task Force on Clinical Practice Guidelines and the Heart Rhythm Society. Circulation. 2018; 138(13): e272-e391, doi: 10.1161/CIR.0000000000000549.

2. Bardy G, Lee K, Mark D, et al. Amiodarone or an Implantable Cardioverter-Defibrillator for Congestive Heart Failure. N Engl J Med. 2005; 352(3): 225-237, doi: 10.1056/nejmoa043399.

3. Moss AJ, Zareba W, Hall WJ, et al. Prophylactic implantation of a defibrillator in patients with myocardial infarction and reduced ejection fraction. N Engl J Med. 2002; 346(12): 877-883, doi: 10.1056/NEJMoa013474, indexed in Pubmed: 11907286.

4. Kalogeropoulos AP, Fonarow GC, Georgiopoulou V, et al. Characteristics and outcomes of adult outpatients with heart failure and improved or recovered ejection fraction. JAMA Cardiol. 2016; 1(5): 510-518, doi: 10.1001/jamacardio.2016.1325, indexed in Pubmed: 27434402.

5. Thomas KL, Al-Khatib SM, Lokhnygina Y, et al. Amiodarone use after acute myocardial infarction complicated by heart failure and/or left ventricular dysfunction may be associated with excess mortality. Am Heart J. 2008; 155(1): 87-93, doi: 10.1016/j. ahj.2007.09.010, indexed in Pubmed: 18082495.

6. Wilcox JE, Fang JC, Margulies KB, et al. Heart Failure with recovered left ventricular ejection fraction: JACC scientific expert panel. J Am Coll Cardiol. 2020; 76(6): 719-734, doi: 10.1016/j. jacc.2020.05.075, indexed in Pubmed: 32762907.

7. Packer DL, Prutkin JM, Hellkamp AS, et al. Impact of implantable cardioverter-defibrillator, amiodarone, and placebo on the mode of death in stable patients with heart failure: analysis from the sudden cardiac death in heart failure trial. Circulation. 2009; 120(22): 2170-2176, doi: 10.1161/CIRCULATIONAHA.109.853689, indexed in Pubmed: 19917887.

8. Rizopoulos DJM. An R package for the joint modelling of longitudinal and time-to-event data. J Statistical Software (Online). 2010; 35(9): 1-33.
9. Rizopoulos D. Joint models for longitudinal and time-to-event data. CRC Press. 2012, doi: 10.1201/b12208.

10. Ibrahim JG, Chu H, Chen LM. Basic concepts and methods for joint models of longitudinal and survival data. J Clin Oncol. 2010; 28(16): 2796-2801, doi: $10.1200 / J C 0.2009 .25 .0654$, indexed in Pubmed: 20439643.

11. Basuray A, French B, Ky B, et al. Heart failure with recovered ejection fraction: clinical description, biomarkers, and outcomes. Circulation. 2014; 129(23): 2380-2387, doi: 10.1161/CIRCULATIONAHA.113.006855, indexed in Pubmed: 24799515.

12. Florea VG, Rector TS, Anand IS, et al. Heart failure with improved ejection fraction: clinical characteristics, correlates of recovery, and survival: results from the valsartan heart failure trial. Circ Heart Fail. 2016; 9(7), doi: 10.1161/CIRCHEARTFAILURE.116.003123, indexed in Pubmed: 27413037.

13. Punnoose LR, Givertz MM, Lewis EF, et al. Heart failure with recovered ejection fraction: a distinct clinical entity. J Card Fail. 2011; 17(7): 527-532, doi: 10.1016/j.cardfail.2011.03.005, indexed in Pubmed: 21703523.

14. Wilcox JE, Fonarow GC, Yancy CW, et al. Factors associated with improvement in ejection fraction in clinical practice among patients with heart failure: findings from IMPROVE HF. Am Heart J. 2012; 163(1): 49-56.e2, doi: 10.1016/j.ahj.2011.10.001, indexed in Pubmed: 22172436.

15. Zhang Y, Guallar E, Blasco-Colmenares E, et al. Changes in follow-up left ventricular ejection fraction associated with outcomes in primary prevention implantable cardioverter-defibrillator and cardiac resynchronization therapy device recipients. J Am Coll Cardiol. 2015; 66(5): 524-531, doi: 10.1016/j.jacc.2015.05.057, indexed in Pubmed: 26227190.

16. Lupón J, Díez-López C, de Antonio M, et al. Recovered heart failure with reduced ejection fraction and outcomes: a prospective study. Eur J Heart Fail. 2017; 19(12): 1615-1623, doi: 10.1002/ ejhf.824, indexed in Pubmed: 28387002.

17. Ghimire A, Fine N, Ezekowitz JA, et al. Frequency, predictors, and prognosis of ejection fraction improvement in heart failure: an echocardiogram-based registry study. Eur Heart J. 2019; 40(26): $2110-2117$, doi: $10.1093 /$ eurhearti/ehz233, indexed in Pubmed: 31280320.

18. Adabag S, Patton KK, Buxton AE, et al. Association of implantable cardioverter defibrillators with survival in patients with and without improved ejection fraction: secondary analysis of the sudden cardiac death in heart failure trial. JAMA Cardiol. 2017; 2(7): 767-774, doi: 10.1001/jamacardio.2017.1413, indexed in Pubmed: 28724134.

19. Kini V, Soufi MK, Deo R, et al. Appropriateness of primary prevention implantable cardioverter-defibrillators at the time of generator replacement: are indications still met? J Am Coll Cardiol. 2014; 63(22): 2388-2394, doi: 10.1016/j.jacc.2014.03.025, indexed in Pubmed: 24727249.

20. Madhavan M, Waks JW, Friedman PA, et al. Outcomes After Implantable Cardioverter-Defibrillator Generator Replacement for Primary Prevention of Sudden Cardiac Death. Circ Arrhythm Electrophysiol. 2016; 9(3): e003283, doi: 10.1161/ CIRCEP.115.003283, indexed in Pubmed: 26921377.

21. Kramer DB, Buxton AE, Zimetbaum PJ. Time for a change a new approach to ICD replacement. N Engl J Med. 2012; 366(4): 291-293, doi: 10.1056/NEJMp1111467, indexed in Pubmed: 22276818.

22. Al-Khatib SM, Friedman DJ, Sanders GD. When is it safe not to reimplant an implantable cardioverter defibrillator at the time of battery depletion? Card Electrophysiol Clin. 2018; 10(1): 137-144, doi: 10.1016/j.ccep.2017.11.014, indexed in Pubmed: 29428135.

23. Naksuk N, Saab A, Li JM, et al. Incidence of appropriate shock in implantable cardioverter-defibrillator patients with improved ejection fraction. J Card Fail. 2013; 19(6): 426-430, doi: 10.1016/j. cardfail.2013.04.007, indexed in Pubmed: 23743493.

24. Schliamser JE, Kadish AH, Subacius H, et al. Significance of follow-up left ventricular ejection fraction measurements in the Defibrillators in Non-Ischemic Cardiomyopathy Treatment Evaluation trial (DEFINITE). Heart Rhythm. 2013; 10(6): 838-846, doi: 10.1016/j.hrthm.2013.02.017, indexed in Pubmed: 23422221. 